\title{
Auditor enfermeiro: visão da equipe de enfermagem
}

\author{
Auditor nurse: vision of the nursing team
}

\author{
1 Guilherme Gontijo Pereira ggontijopereira@bol.com.br \\ 1 Thainá Nascimento Roly \\ 1 Clarice Mayremi Toshimitu Hoyashi \\ 1 Lucrecia Helena Loureiro
}

1 Centro Universitário de Volta Redonda - UniFOA

\section{Resumo}

O enfermeiro que exerce a função assistencial em seu cotidiano é responsável por observar, avaliar, determinar e executar as ações prestadas ao paciente. Nesse sentido, seu trabalho visa verificar e controlar os custos operacionais, concomitantes ou não à realização de ações assistenciais, garantindo a qualidade, eficiência e eficácia nas instituições de saúde. Nesse contexto, o estudo tem como objetivo a compreensão do trabaIho do auditor enfermeiro pela equipe de enfermagem hospitalar; descrever o papel do auditor enfermeiro nas instituições hospitalares no entendimento da equipe de enfermagem; e apontar os registros de enfermagem considerados imprescindíveis no prontuário. Trata-se de um estudo de campo, descritivo, exploratório de caráter qualitativo. A pesquisa foi realizada após aprovação do Comitê de Ética- CoEPS. Os sujeitos do estudo foram 30 enfermeiros e 20 técnicos de enfermagem de dois hospitais da região do Médio Paraíba. 0 período do estudo aconteceu entre fevereiro e outubro de 2018. 0 instrumento da coleta de dados foi um questionário de perguntas abertas e os resultados puderam evidenciar que a maioria dos enfermeiros e técnicos de enfermagem conhecem o papel do auditor enfermeiro, valorizaram os registros de cuidados de enfermagem e os materiais gastos com essa prática, o que reflete no faturamento hospitalar. As orientações procedentes do auditor enfermeiro são construtivas e bem aceitas pela equipe de enfermagem.

\section{Palavras-chave:}

Auditoria de enfermagem. Prontuário. Registros de enfermagem.

\begin{abstract}
Nurses who perform the care function, in their daily lives, are responsible for observing, evaluating, determining and performing assistance rendered to the patient. In this sense, this work aims to verify and control the operational costs, concomitant or not, to carry out assistance actions, guaranteeing the quality, efficiency and effectiveness in health institutions. In this context, the study aims to understand the work of the nursing auditor regarding its appreciation by the hospital nursing team; describe the role of the nursing auditor in the hospital institutions as understood by the nursing team, pointing out the nursing records considered essential in the medical record. This is a descriptive, exploratory field study of qualitative nature. The research was carried out after approval of the Ethics Committee - CoEPS. The subjects of the study were 30 nurses and 20 nursing technicians from two hospitals in the region of Medium Paraíba Valley. The study period was between February and October 2018. The data collection instrument was an openended questionnaire and the results showed that the majority of nurses and nursing technicians are aware of the role of the nursing auditor, they value the nursing care records and materials spent with that practice, which reflects in the hospital billing. The guidelines coming from the nursing auditor are constructive and well accepted by the nursing team.
\end{abstract}

\section{Keywords:}

Nursing auditor. Record. Nursing records.

\section{Como você deve citar?}

PEREIRA, Guilherme Gontijo et al. Auditor enfermeiro: visão da equipe de enfermagem. Cadernos UniFOA, Volta Redonda, n. 40, p. 107-116, agosto. 2019. 


\section{INTRODUÇÃO}

Com as constantes inovações tecnológicas, frutos do desenvolvimento humano e da criatividade em busca da qualidade nos serviços de saúde, especialistas em gestão ampliam as estratégias de controle, regulação, avaliação e auditoria nos munícipios, a fim de monitorar seus processos de trabalho.

Luongo (2011) define a auditoria como um exame sistemático e independente dos atos e decisões das pessoas em relação à qualidade, com o objetivo de determinar as atividades realizadas de acordo com as exigências das operadoras, planejando e implementando para alcançar o objetivo proposto.

A equipe de auditores é constituída por diversos profissionais de saúde capacitados de diferentes áreas de atuação, entretanto este estudo enfoca a visão da equipe de enfermagem sobre o trabalho do auditor enfermeiro. Na ótica da auditoria, a redução de custos na saúde consiste no controle da realização e registros dos procedimentos médicos, sendo esses instrumentos de análises de um auditor. Para exercer essa função, vislumbram-se profissionais de saúde capacitados para analisar documentos e realizar observações in loco.

O enfermeiro é um dos profissionais da área da saúde que possui conhecimento específico em várias áreas de sua atuação. 0 conhecimento teórico e prático adquirido em curso de auditoria possibilita que esse profissional exerça a função de auditor.

De acordo com a Resolução - Anexo 266/2001 (COFEN, 2001), é papel do Auditor Enfermeiro, em seu exercício, ter visão holística, como a qualidade de gestão, qualidade de assistência e quântico-econômico-financeiro, tendo em vista sempre o bem-estar do ser humano enquanto paciente.

Vale ressaltar que a assistência da equipe multidisciplinar durante a hospitalização do paciente requer uma avaliação criteriosa de um profissional capacitado para identificar problemas relativos aos cuidados, diferente da assistência prestada em nível ambulatorial.

Para Luongo (2011), quando os problemas são previamente identificados pelo auditor, transtornos futuros poderão ser evitados. Uma intervenção imediata possibilitará corrigir e evitar danos ao paciente ou prejuízos às instituições.

Nesse raciocínio, a motivação em realizar uma pesquisa com essa temática foi devido ao fato de que o auditor enfermeiro colabora com a melhoria da gestão hospitalar, controla os gastos e auxilia na redução de custos operacionais, uma preocupação incipiente de muitos profissionais assistenciais. Mas a inquietação vai além das funções de um enfermeiro auditor e, por isso o estudo visa investigar o que pensam os colaboradores de enfermagem, principalmente acerca desses profissionais da prática hospitalar.

As ferramentas de trabalho de um auditor são os registros da equipe multidisciplinar envolvida no processo do cuidar dos pacientes internados, que possibilita avaliar a qualidade da assistência da admissão à alta.

De acordo com o Ministério da Saúde, no Manual de Auditoria do Sistema Único de Saúde (SUS) (BRASIL 2011), foram descritas as finalidades da Auditoria e tem-se o seguinte item entre outras: "aferir a preservação dos padrões estabelecidos e proceder ao levantamento de dados que permitam conhecer a qualidade, a quantidade, os custos e os gastos da atenção à saúde". 
À luz dos hospitais privados, diferentemente dos hospitais públicos, há uma grande diferença no que diz respeito à forma de remuneração dos custos assistenciais, utilizando-se tabelas de preços distintos baseados nos contratos. Tal fato leva os auditores a serem capacitados a analisar contratos, as tabelas de valores dos procedimentos, materiais, serviços e o manual relativos à auditoria na rede privada e pública.

Baseando-se nos registros dos prontuários, a história de internação dos pacientes deve conter relatos dos profissionais que os assistiram, com o detalhamento do quadro clínico, e pela equipe de enfermagem, cuidados de enfermagem prescritos e implementados, assim como, as intervenções de outros profissionais de saúde.

O relato encontrado no prontuário do paciente não só contribui para norteio do tratamento, mas também para dar visibilidade em que condições o paciente foi hospitalizado. Assim como o conteúdo escrito, evidenciado e autorizado pela auditoria possibilitará o ressarcimento dos custos desse atendimento.

Segundo Potter (2005), o prontuário médico, no qual constam os registros de cada paciente sobre a história clínica, exames laboratoriais e resultados de estudos diagnósticos e o plano de tratamento proposto também possui as respostas das ações planejadas e executadas pela equipe multidisciplinar de saúde. Essas informações são confidenciais, mas são compartilhadas entre os prestadores de cuidados.

Os auditores enfermeiros, juntamente com os auditores médicos, são profissionais capacitados a analisar os registros realizados por seus pares, cada qual dentro de seus conhecimentos específicos da sua prática profissional, baseando-se nos princípios científicos e éticos.

A problemática do estudo está relacionada com o cotidiano das práticas de enfermagem, em que as ações de cuidados são intensamente desenvolvidas para promoção do bem-estar dos pacientes hospitalizados. Entretanto, as informações dos pacientes necessitam ser detalhadas, pois servirão para que auditores e magistrados possam avaliar a qualidade assistencial durante a hospitalização. Isso porque, no caso de eventual demanda judicial, as informações prestadas pelas equipes médicas e de enfermagem podem servir como prova documental suplementar para subsidiar a tomada de decisões pelo magistrado do feito. Sobre isso, Altoé (2017) descreve que é comum demanda judicial no cotidiano dos hospitais públicos e privados, quando os gestores se deparam com solicitações de prontuários médicos encaminhados por magistrados.

O prontuário é definido pelo Conselho Federal de Medicina (Resolução $n^{\circ}$ 1.638/2002) como documento único constituído de um conjunto de informações, sinais e imagens registradas, geradas a partir de fatos, acontecimentos e situações sobre a saúde do indivíduo.

Vale ressaltar a importância e o significado do prontuário como instrumento de comunicação entre a equipe assistencial, também de caráter legal, como estabelecido pelo Conselho Federal de Medicina.

Para ser compartilhado entre os profissionais de saúde e a área jurídica, quando necessário, o prontuário necessita ter registros que sejam legíveis, concisos e de fácil interpretação, além de ordenado cronologicamente, facilitando a compreensão dos dados escritos.

Na prática, pode-se entender que a gestão de qualidade se desmembra em gestão de cuidados e gestão administrativa ou financeira. Tanto uma quanto a outra funcionam como uma engrenagem, com a finalidade de proporcionar a melhor assistência à saúde, prevendo investimentos que mantenham as instituições de saúde competitivas no mercado. 
De acordo com Silva (2015), alguns estudos apontam que a auditoria permite uma avaliação dos aspectos quanti-qualitativos relacionados à assistência, englobando também a observação sob o ponto de vista organizacional, operacional e financeiro, mas sempre com o foco da qualidade dos cuidados prestados. Assim é coerente dizer que o prontuário do paciente com os registros devem ser o foco de atenção, principalmente para garantir o recebimento do valor gasto na prestação de cuidados, tendo em vista que é um documento legal.

Sobre a qualidade dos prontuários, destaca-se a necessidade do preenchimento correto de todas as informações e dados sobre o paciente. A maioria dos registros são realizados pela enfermagem, pelo fato de estarem durante 24 horas na assistência. Sendo assim, foram traçados os seguintes objetivos para pesquisa: descrever o papel do auditor enfermeiro; apontar os registros de enfermagem considerados imprescindíveis no prontuário do paciente e descrever qual o impacto das orientações recebidas da auditoria.

Acredita-se que o estudo poderá contribuir para melhorar o conhecimento de acadêmicos de enfermagem no contexto da auditoria e conhecer a atuação, dificuldades e desafios a serem enfrentados no dia a dia do auditor enfermeiro. Na prática, poderá levar a equipe de enfermagem a repensar as atividades de registro, dando maior significado à comunicação escrita entre os profissionais de saúde e, dar visibilidade à qualidade assistencial prestada ao paciente, além de possibilitar a redução de custos. Espera-se que a pesquisa possa servir de referência para novos estudos envolvendo à temática.

Foram traçados os seguintes objetivos: descrever o papel do auditor enfermeiro; apontar os registros de enfermagem considerados imprescindíveis no prontuário do paciente e descrever qual o impacto das orientações recebidas da auditoria.

\section{MATERIAIS E MÉTODOS}

Trata-se de um estudo de campo, descritivo exploratório de caráter qualitativo, que requer uma investigação sobre a visão da equipe de enfermagem sobre o trabalho do auditor enfermeiro.

De acordo com Figueiredo (2009), a pesquisa qualitativa surge diante da impossibilidade de investigar e compreender, por meio de dados estatísticos, alguns fenômenos voltados para a percepção, intuição e subjetividade.

Segundo Maria (2002), a pesquisa traz a necessidade do diálogo com a realidade que se pretende investigar e com o diferente, um diálogo enriquecido de críticas, dando significados aos momentos criativos.

A pesquisa foi aplicada em dois hospitais de médio porte, sendo um público com 110 leitos e outro privado com 73 leitos, localizados respectivamente nos municípios de Volta Redonda (RJ) e Barra Mansa (RJ), entre os meses de fevereiro a outubro de 2018.

A amostra dos sujeitos do estudo foi enfermeiros e técnicos de enfermagem, escolhidos aleatoriamente, sendo constituído por 25 profissionais de enfermagem de cada instituição. Desses, foram investigados 30 enfermeiros e 20 técnicos de enfermagem.

Os critérios de inclusão foram os técnicos de enfermagem e enfermeiros de plantão no período da pesquisa e os critérios de exclusão foram técnicos de enfermagem e enfermeiros que no período da 
pesquisa estavam de folga, férias, licença ou que não concordaram em assinar o Termo de Consentimento Livre e Esclarecido.

$\mathrm{O}$ instrumento de coleta de dados proposto foi um questionário de perguntas abertas, aprovado pelo CoEPS (Comitê de Ética em Pesquisa em Seres Humanos) do UniFOA, sob o Parecer No 2.761.543. Os questionários foram aplicados pelos pesquisadores.

Os sujeitos da pesquisa que aceitaram participar assinaram o Termo de Consentimento Livre e Esclarecido, conforme Conselho Nacional de Saúde, resolução 196/96, de pesquisa envolvendo seres humanos.

Após o retorno dos questionários, foram identificados aleatoriamente por categoria profissional: "Enf.A" e "Téc.A", para enfermeiros e técnicos de enfermagem do hospital público, "Enf. B" e "Téc. B", para enfermeiros e técnicos de enfermagem do hospital privado, acompanhados de sequência numérica.

Após leitura interpretativa dos questionários, as respostas foram agrupadas por categorias similares, a fim de facilitar a discussão dos resultados.

\section{RESULTADOS E DISCUSSÃO}

A primeira investigação no estudo consistiu em descrever o papel do auditor enfermeiro nas instituições hospitalares no entendimento da equipe de enfermagem. Verificou-se que 30 (100\%) enfermeiros, sendo 15 do hospital público e 15 do hospital privado, e 20(100\%) técnicos de enfermagem, 10 profissionais de cada instituição, apontaram as principais funções do auditor enfermeiro como: a) profissional que analisa os prontuários para conferir o quadro clínico do paciente justificando a internação, assim como a qualidade assistencial e b) custos operacionais, formando duas categorias:

\section{Categoria 1 - Qualidade Assistencial: auditoria em prontuários}

Análise dos prontuários dos pacientes pós alta hospitalar tem a finalidade de verificar o histórico do atendimento e possibilita identificar registros que mostram a qualidade da assistência prestada no período de hospitalização. Nesse sentido, 21 (70\%) enfermeiros e 12 (60\%) técnicos descreveram que a qualidade dos registros nos prontuários expõe também a qualidade assistencial, possibilitando contribuir para o processo de melhorias nos registros de enfermagem e a prestação dos cuidados de enfermagem, conforme fala dos depoentes:

\footnotetext{
Monitorar através da avaliação do prontuário do paciente processos de qualidade utiliza-se também protocolos operacionais padrão para fazer este monitoramento. Enf.A 1. (Sic)
}

Avaliação sistemática da qualidade da assistência de enfermagem prestada ao cliente e contribui para melhorar a qualidade do serviço prestado através da análise de prontuário. Garantir cobrança adequada. Enf. B 25. (Sic)

O prontuário do paciente é um instrumento de registro da história da doença, tratamento e cuidados implementados pela equipe multidisciplinar de saúde. Nesse registro, é possível avaliar a qualidade da assistência prestada por cada profissional, se as necessidades foram atendidas e se o tratamento foi adequado ou não durante a hospitalização. Além disso, os registros dos prontuários servem como instrumento jurídico, quando necessário. 
Para Barral (2012), os registros de enfermagem são uma forma de comunicação escrita de informações do cliente e dos cuidados prestados pela equipe de enfermagem. São essenciais na documentação do cuidado e, quando redigidos adequadamente, estabelecem uma comunicação efetiva entre a equipe de enfermagem e os demais profissionais envolvidos na assistência, favorecendo a avaliação dos cuidados pela auditoria de enfermagem, além de colaborar com o ensino e pesquisa em enfermagem.

\section{Categoria 2 - Controle de custos assistenciais: o papel do auditor enfermeiro}

No entendimento de 8 (27\%) enfermeiros e 4 (20\%) técnicos de enfermagem, o auditor enfermeiro desenvolve a atividade de analisar no prontuário a cobrança de procedimentos, medicamentos e insumos gastos no atendimento ao paciente durante $o$ atendimento hospitalar, de maneira a evitar pagamentos indevidos e controlar custos para operadoras de saúde ou SUS, no sistema público de saúde. Verificar o porquê, valor gasto, as perdas e onde foram gastos os materiais e/ou insumos, em determinados
procedimentos/pacientes (enf. A 6).

Minimizar desperdícios de materiais, medicamentos, utilização errada de equipamentos, bem como de recursos humanos e revisão de glosas. Com isso, avaliar a qualidade da assistência de enfermagem e os custos decorrentes da prestação desta atividade (enf. B 22).

As anotações de enfermagem, segundo Pellegrini (2004), são, em sua maioria, inconsistentes ou ilegíveis e a glosa tem sido cada vez mais frequente nas instituições de saúde. Glosar significa o cancelamento ou indeferimento parcial ou total de orçamento, conta ou verba por serem considerados ilegais ou indevidos, ou seja, refere-se aos itens que o auditor da operadora (plano de saúde) não considera adequado para pagamento.

Nesse raciocínio, a prática do cotidiano, na visão do auditor enfermeiro, deve ser revista, porque a perda de faturamento impacta no prejuízo às organizações mantenedoras. Além disso, um registro deficiente de informações pode desqualificar a assistência prestada e a credibilidade da instituição comprometida.

Os resultados mostraram que a maioria dos enfermeiros possui conhecimentos sobre a atuação do auditor enfermeiro em suas instituições de saúde. Apenas 1(3\%) enfermeiro respondeu não ter conhecimento e $4(20 \%)$ técnicos de enfermagem, não souberam dizer a atividade desempenhada pelo auditor enfermeiro. Conforme dados da pesquisa.

Não sei (enf. A 15).

Não tem como opinar, trabalho na rede pública e desconheço a existência desse tipo de profissional (téc. A 22).

De acordo com Silva et al. (2016), o auditor enfermeiro avalia a assistência de enfermagem utilizando como instrumento de seu trabalho o prontuário, possibilitando, em sua análise, o acompanhamento do cliente e verificando a compatibilidade entre o procedimento realizado e os itens que compõem a conta hospitalar cobrada, deferindo o pagamento justo mediante a cobrança adequada.

Evidenciou-se, por meio das respostas dos enfermeiros lotados tanto em hospital público quanto em hospital privado, um entendimento adequado sobre o papel do auditor enfermeiro em instituições de saúde, compatíveis com as literaturas.

Na visão de enfermeiros e técnicos de enfermagem, os registros necessários a serem descritos nos prontuários dizem respeito à realização de cuidados de enfermagem e os itens gastos na assistência. 
Nesse raciocínio, 30 (100\%) enfermeiros e 19 (95\%) técnicos de enfermagem, sendo 15 enfermeiros de cada hospital, 10 técnicos de enfermagem da instituição privada e 9 técnicos de enfermagem da instituição pública, afırmaram que são imprescindíveis tais registros.

\section{Categoria 3 - Registros de enfermagem: um olhar para o cuidado.}

Os enfermeiros citaram no estudo que o registro imprescindível no prontuário do paciente é toda a assistência prestada durante a hospitalização. A maioria dos enfermeiros $26(87 \%)$ afırmou que os registros de enfermagem respaldam o profissional mediante ao seu trabalho assistencial, fazendo-se presente aos cuidados de enfermagem e as possíveis intercorrências que houver durante a hospitalização.

Tudo que acontece com o paciente deve ser registrado e considerado importante, estado geral, exames realizados, anamnese, exame físico, evento adverso, aceitação da dieta, alterações de humor, ausência de visita, entre outros (enf. A 4).

Acredito na análise das evoluções tanto médica quanto da enfermagem em nível de supervisão e pessoal de apoio (téc. A 22).

Segundo o COFEN (2016), é fundamental a existência dos registros de enfermagem no processo de cuidar. Desse modo, quando são relatados detalhadamente, retratam a realidade documentada, facilitam a comunicação entre a equipe, auxilia na troca de plantão, além de contribuir para outras finalidades relacionadas ao paciente, tais como: ensino, auditorias, processos jurídicos, planejamento e prevenção, fins estatísticos, acompanhamento do cuidado prestado.

No exercício que compete ao enfermeiro, torna-se oportuno o registro de enfermagem contendo as informações específicas do estado e evolução do paciente, conscientizando-se sobre a importância da anotação, quando se trata de respaldo legal, acompanhamento do cliente e relatos de gastos para controle do faturamento.

\section{Categoria 4 - Gastos na assistência de enfermagem: foco no registro}

Em toda assistência ofertada ao paciente, está inserido o gasto com medicamentos e insumos, pois assim como todo tratamento que envolve a equipe multidisciplinar de saúde possui custos assistenciais. Sendo assim, 4 (13\%) enfermeiros enfatizaram a necessidade de detalhar os cuidados de enfermagem, checagem de procedimentos realizados e a identificação do profissional executante. Dessa maneira, garante-se uma fatura de contas adequada à prestação de serviços.

Todos os materiais gastos e quanto tempo foi utilizado (enf. A 6).

Deve ter compatibilidade entre o procedimento realizado, o lançamento adequado dos materiais e os itens que compõem a conta hospitalar, garantindo assim, uma cobrança adequada (enf. B 25).

Rodrigues et al. (apud BRAGAS, 2015) descreveram que os registros de enfermagem vinculados ao prontuário do paciente também são usados para o faturamento e cobrança, na auditoria interna ou externa para obtenção de dados sobre as atividades realizadas e para análise institucional. Boa parte do ressarcimento de materiais, medicamentos, procedimentos e outros serviços estão vinculados à anotação de enfermagem.

Com a mesma ideia de Bragas (2015), Amaral et al. (2016) confirmam que o exercício da auditoria começou a ser visualizado como um instrumento que regula a parte financeira e, sendo assim, permite 
aos gestores distinguir os processos de custos operacionais relacionados aos lucros e despesas, em que uma delas são as glosas pelas anotações de enfermagem.

Com a pesquisa, verificou-se que em ambas as instituições, pública e privada, a maioria dos enfermeiros enfatizou que os registros imprescindíveis são os relatos sobre os cuidados de enfermagem. Nessa vertente, há um indicativo que os profissionais que realizam os registros também devem entender que os ressarcimentos dos custos assistenciais estão atrelados ao detalhamento das anotações de enfermagem. Dessa maneira, a auditoria consegue realizar a cobrança adequada evitando glosas.

No que tange à aceitação de profissionais de enfermagem quanto às orientações provenientes do auditor enfermeiro nas instituições de saúde, 30 (100\%) enfermeiros e 17(85\%) técnicos de enfermagem descreveram que há aceitação das orientações do auditor enfermeiro como crítica construtiva, formando categoria única. A função do auditor enfermeiro é orientadora e não punitiva.

Entretanto, 2(10\%) técnicos de enfermagem disseram que nenhuma conduta foi orientada proveniente do auditor enfermeiro no setor em que atua e, se houvesse qualquer orientação, seria bem aceito.

No estudo, apenas 1 (5\%) técnico de enfermagem não respondeu ao questionamento.

É relevante citar que o auditor enfermeiro das instituições sensibilize enfermeiros das várias unidades de internação para a realização de registros adequados no prontuário do paciente. Porém, não se sabe o porquê de esses dois técnicos de enfermagem nunca receberam orientações direta ou indiretamente da chefia imediata de enfermagem.

\section{Categoria 5 - Educação em Serviço: o auditor enfermeiro em destaque}

De acordo com os depoimentos, a maioria reconhece o auditor enfermeiro como orientador de ações corretivas no processo de registros dos prontuários que favorece a melhoria do seu trabalho.

Recebo bem, pois sei que o trabalho é importante para o faturamento hospitalar (enf. A 7).

Entende-se que críticas, orientações, recomendações, elogios são construtivos, e para uma melhora do funcionamento do setor (téc. A 16).

Acho que somos uma equipe e, quando se é dado uma orientação, é para o bem de todos, principalmente para o paciente e para o andamento hospitalar (téc. B 1).

Para Pinto e Melo (2010), o enfermeiro pode atuar sugerindo mudanças de rotinas organizacionais e subsidiar a educação continuada com o enfoque na prestação de serviços de qualidade.

A educação continuada é uma capacitação e/ou aperfeiçoamento do profissional para as atividades e consiste em manter os colaboradores sempre em um constante processo educativo. Nesse âmbito, o auditor enfermeiro irá, mediante as dificuldades encontradas no período de análise dos prontuários, identificar qual é a parte que mais se encontra defasada e capacitará a equipe com o objetivo de melhorar o serviço desempenhado.

Na atuação do auditor enfermeiro, uma das ações é a orientação explícita à equipe multidisciplinar de saúde que presta assistência direta ao paciente, sendo, no caso da enfermagem, o enfermeiro auditor responsável por transferir tal ação aos enfermeiros dos setores de trabalho. 
Em geral, na prática, o auditor solicita aos seus colaboradores a melhoria dos relatos assistenciais de maneira detalhada, com a escrita legível, a frequência regular de anotações com data e identificação correta dos profissionais executores, a quantificação de materiais, medicamentos e tempo de utilização de gases medicinais, no período de hospitalização.

\section{CONCLUSÃO}

0 auditor enfermeiro executa diversas atividades administrativas gerenciais, incluindo a análise de contas hospitalares. Essa análise, por sua vez, consiste na garantia de pagamento adequado aos custos operacionais da assistência prestada aos pacientes durante a hospitalização.

Os resultados do estudo puderam evidenciar que a maioria dos enfermeiros e técnicos de enfermagem conhece o papel do auditor enfermeiro e tem boa aceitação das suas orientações para a melhoria do serviço e o faturamento hospitalar.

Os apontamentos descritos pela equipe de enfermagem sobre os registros imprescindíveis no prontuário do paciente relacionaram-se com os cuidados de enfermagem e os itens gastos na assistência prestada.

A pesquisa possibilitou concluir que os profissionais de enfermagem, por possuírem conhecimento a respeito da finalidade proposta pelos profissionais auditores, demonstraram estar preparados para as sugestões procedentes da auditoria em suas instituições.

Embora tenham sido satisfatórios os resultados do estudo implementado em duas instituições hospitalares, analisando a atuação do enfermeiro auditor na visão da equipe de enfermagem, esta pesquisa consiste apenas em um estudo entre vários outros que poderão ser realizados sobre a temática, para dar visibilidade ao auditor enfermeiro e os aspectos sobre o seu trabalho na saúde.

\section{REFERÊNCIAS}

ALTOÉ, F. M. F. Do fornecimento de prontuários médicos depositados nas instituições de saúde quando requisitados pelas autoridades públicas. Agosto, 2017. Disponível em: https://jus.com.br/artigos/60113/. Acesso em: 17 mar. 2018.

AMARAL, R. C.; ABREU, A. M.; FEIJÓ, E. J.; VALE, C. W. F.; ANDRADE, N. C. Importância do registro de enfermagem para o faturamento hospitalar. revisão da literatura. Revista Universo, v.1, n. 1, p.247-263, 2016.

BARRAL, L. N. M. et al. Análise dos registros de enfermagem em prontuários de pacientes em um Hospital de Ensino. Revista Mineira de Enfermagem. v. 16, n.2, 2012.

BRASIL. Ministério da Saúde. Auditoria do SUS - Orientações Básicas. Brasília (DF), 2011. p. 17. http:// sna.saude.gov.br/download/LivroAuditoriaSUS_14x21cm.pdf. Acesso em: 13 jun. 2017.

CONSELHO FEDERAL DE ENFERMAGEM (Brasil). Resolução COFEN - 266/2001. Anexo. Rio de Janeiro, 05 de outubro de 2001. Disponível:http://www.cofen.gov.br/resoluo-cofen-2662001_4303.html. Acesso em: 17 mar. 2018. 
CONSELHO FEDERAL DE ENFERMAGEM (Brasil). Guia de recomendações para registros de enfermagem no prontuário do paciente e outros documentos de enfermagem, 2016. Disponível: http://www.cofen. gov.br/wp-content/uploads. Acesso em: 01 set. 2018.

CONSELHO FEDERAL DE MEDICINA (Brasil). Resolução CFM n ${ }^{1.638 / 2002 . ~ D e f i n e ~ p r o n t u a ́ r i o ~ m e ́ d i c o, ~}$ 2002, Seção I, p. 184-5. Disponível em: http://www.portalmedico.org.br/resolucoes/cfm/2002/1638_2002. htm. Acesso em: 17 mar. 2018.

FIGUEIREDO, N. M. Método e metodologia na pesquisa científica. 3. ed. São Caetano do Sul, SP. Bendiz Editora, 2009.

FIGUEIREDO, N. M. A.; MACHADO, W. C. A. (org.). Tratado de cuidados de enfermagem médico-cirúrgico. Volume I. São Paulo: Roca, 2012.

GONÇALVES, E. P. Iniciação científica. 2001. Disponível em: http://books.scielo.org/id/vwc8g/pdf/ piana-9788579830389-06.pdf. Acesso em: 30 abr. 2018.

MARIA, J. F. A família como espaço privilegiado para a construção da cidadania. Franca, São Paulo, Brasil. 2002. Dissertação- UNESP, FHDSS, São Paulo, 2002.

LUONGO, J. Gestão de qualidade em saúde. São Paulo: Editor Fidel, 2011.

PELLEGRINI, G. Glosas convênio x prestador. Anais do Congresso Latino Americano de Serviços de Saúde e 3a Jornada de Gestão e Clínicas Médicas. São Paulo; 2004.

PINTO, K. A.; MELO, C. M. M. A prática da enfermeira em auditoria em saúde. Rev. esc. enferma. USP [online]. São Paulo, v. 44, n. 3, p. 671-678, 2010.

POTTER, P. A.; PERRY, A. G. Fundamentos de Enfermagem. 6. ed. Rio de Janeiro: Elzevir, 2005.

RODRIGUES, V. A; PERROCA, M. G.; JERICÓ, M. C. Glosas hospitalares: importância das anotações de enfermagem. Arq Ciênc Saúde 2004; v.11, n.4; out-dez. 2004.

SILVA, J. S. Auditoria em saúde: um novo paradigma na qualidade da assistência de enfermagem. Tradução em Português. Rev Enferm UFPI. 2015 Apr-Jun; 4(2):130-4.

SILVA, K. R.; LIMA, M. D. O.; SOUSA, M. A. Auditoria: ferramenta de enfermagem para melhoria da qualidade assistencial. Revista Eletrônica Gestão \& Saúde .v.07, n. 02,.p 793, 2016. 